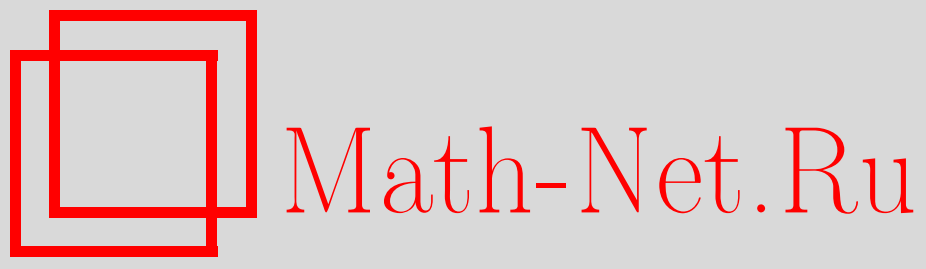

Е. Д. Лившиц, О непрерывном почти наилучшем приближении функций в пространстве $C[0,1]$, Функи. анализ и его прил., 2001, том 35, выпуск $1,85-87$

DOI: https://doi.org/10.4213/faa236

Использование Общероссийского математического портала MathNet.Ru подразумевает, что вы прочитали и согласны с пользовательским соглашением

http://www . mathnet.ru/rus/agreement

Параметры загрузки:

IP : 35.173 .219 .149

26 апреля 2023 г., 16:56:34

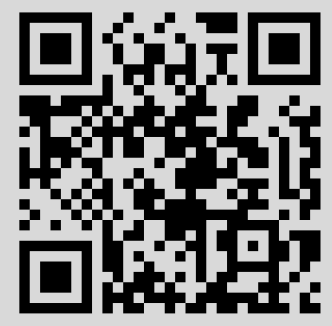


УДК 517.9

\title{
О непрерывном почти наилучшем приближении функций в пространстве $C[0,1]$
}

\author{
(c) 2001. Е. Д. Лившиц
}

Рассматривается задача о почти наилучшем приближении функций в пространстве $C[0,1]$. Пусть $\varepsilon>0$. Напомним, что оператором почти наилучшего приближения, или $\varepsilon$-проекциией элемента $x \in C[0,1]$ на множество $L \subset C[0,1]$, называется многозначное отображение

$$
x \mapsto P_{L, \varepsilon}(x)=\{z \in L:\|x-z\| \leqslant \rho(x, L)+\varepsilon\},
$$

где $\rho(x, L)=\inf _{y \in L}\|x-y\|-$ расстояние от $x$ до $L$. При $\varepsilon=0$ оператор $P_{L, \varepsilon}$ является оператором метрической проекции. Отображение $G: x \mapsto z \in P_{L, \varepsilon}$ называется $\varepsilon$-выборкой (из оператора почти наилучшего приближения); в случае его непрерывности $G$ называют непрерывной $\varepsilon$-выборкой.

В 1859 г. П. Л. Чебышёв [1] отметил, что множество $R_{m, n}$ алгебраических рациональных дробей $R_{m, n}=\left\{\frac{a_{m} x^{m}+\cdots+a_{0}}{b_{n} x^{n}+\cdots+b_{0}}, a_{i}, b_{j} \in \mathbb{R}\right\}$ является множеством существования и единственности (как говорят сейчас, чебышёвским множеством) в $C[0,1]$. Мэли и Вицгилем [3] было показано, что метрическая проекция на $R_{m, n}$ разрывна. Тем не менее, как установил С. В. Конягин [2], для любого $\varepsilon>0$ на $R_{m, n}$ существует непрерывная $\varepsilon$-выборка.

Ниже будет показано, что непрерывная $\varepsilon$-выборка на множество $L$ существует в случае, когда $L$ принадлежит некоторому классу множеств, включающему в себя, в частности, множество алгебраических рациональных дробей и множество кусочно-линейных функций.

Пусть $n \geqslant 2, m \geqslant 0$. Рассмотрим непустое открытое множество $D \subset[0,1]^{n-1}$ и множество $L=\operatorname{Im} F$, где $F: D \times \mathbb{R}^{m+1} \rightarrow C[0,1]-$ некоторое непрерывное и инъективное отображение.

Для произвольного $\varepsilon>0$ рассмотрим отображение $Y_{\varepsilon, L}: C[0,1] \times D \rightarrow 2^{\mathbb{R}^{m+1}}$, положив для $f \in C[0,1]$ и $\left(x_{1}, \ldots, x_{n-1}\right) \in D$

$$
\begin{aligned}
& Y_{\varepsilon, L}\left(f, x_{1}, \ldots, x_{n-1}\right) \\
& \quad=\left\{\left(y_{0}, \ldots, y_{m}\right):\left\|F\left(x_{1}, \ldots, x_{n-1}, y_{0}, \ldots, y_{m}\right)-f\right\|<\rho(f, L)+\varepsilon\right\} .
\end{aligned}
$$

Определим $K$ как класс множеств $L$, удовлетворяющих следующим условиям:

(i) для любых $\varepsilon>0$, функции $f \in C[0,1]$ и набора $\left(x_{1}, \ldots, x_{n-1}\right) \in D$ множество $Y_{\varepsilon, L}\left(f, x_{1}, \ldots, x_{n-1}\right)$ ограничено и выпукло;

(ii) для произвольных $f \in C[0,1], \varepsilon>0$ и целого $k, 0 \leqslant k \leqslant n-2$, при любых действительных $x_{1}, \ldots, x_{k}$ множество, состоящее из таких $x_{k+1}$, что найдутся $\left(x_{k+2}, \ldots, x_{n-1}\right)$, при которых $\left(x_{1}, \ldots, x_{n-1}\right) \in D, Y_{\varepsilon, L}\left(f, x_{1}, \ldots, x_{n-1}\right) \neq \varnothing$, связно в $\mathbb{R}$ (пустое множество считается связным);

(iii) отображение $Y_{\varepsilon, L}$ непрерывно во всех точках, где $Y_{\varepsilon, L}\left(f, x_{1}, \ldots, x_{n-1}\right) \neq \varnothing$ (в образе рассматривается хаусдорфова метрика).

Tеорема 1. Пусть $L \in K u \varepsilon>0 ;$ тогда существует отображение $G \in$ $C(C[0,1], L)$ (непрерывная $\varepsilon$-выборка), такое, что для любой функции $f \in$ 
$C[0,1]$ справедливо неравенство

$$
\|G(f)-f\| \leqslant \rho(f, L)+\varepsilon .
$$

ДокАЗАТЕЛЬСтво. Пусть $\mu-$ лебегова мера на пространстве $\mathbb{R}^{m+1}$. Рассмотрим строго монотонную функцию $\varphi \in C\left(\mathbb{R}_{+},[0,1]\right)$, такую, что $\varphi(0)=0$. Для любой функции $f \in C[0,1]$ положим

$$
V_{f}\left(x_{1}, \ldots, x_{n-1}\right)= \begin{cases}\varphi\left(\mu\left(Y_{\varepsilon, L}\left(f, x_{1}, \ldots, x_{n-1}\right)\right)\right), & \left(x_{1}, \ldots, x_{n-1}\right) \in D, \\ 0, & \left(x_{1}, \ldots, x_{n-1}\right) \notin D .\end{cases}
$$

Определение функции $V_{f}$ корректно в силу условия (i).

Укажем способ построения непрерывной $\varepsilon$-выборки $G$. В силу того что $L-$ образ непрерывного и инъективного отображения, топология в $L$, индуцируемая евклидовой нормой в $D \times \mathbb{R}^{m+1}$, не слабее топологии в $L$, порожденной равномерной нормой. Поэтому при любых $f \in C[0,1]$ и $\left(x_{1}, \ldots, x_{n-1}\right) \in D$ множество $Y_{\varepsilon, L}\left(f, x_{1}, \ldots, x_{n-1}\right)$ открыто, и, следовательно, если $Y_{\varepsilon, L}\left(f, x_{1}, \ldots, x_{n-1}\right)$ непусто, то $V_{f}\left(x_{1}, \ldots, x_{n-1}\right)>0$. Пусть $f \in C[0,1]$. Из условия (ii) нетрудно вывести, что множество тех наборов $\left(x_{1}, \ldots, x_{n-1}\right) \in \mathbb{R}^{n-1}$, для которых $V_{f}\left(x_{1}, \ldots, x_{n-1}\right)>0$, измеримо; по условию (iii) функция $V_{f}$ непрерывна на этом множестве и, следовательно, интегрируема на $[0,1]^{n-1}$. Последовательно определим отображение $X_{i}(f): C[0,1] \rightarrow[0,1](1 \leqslant i \leqslant n-1)$ следующим образом:

$$
\begin{aligned}
& \int_{0}^{X_{i}(f)} \int_{0}^{1} \ldots \int_{0}^{1} V_{f}\left(X_{1}(f), \ldots, X_{i-1}(f), x_{i}, \ldots, x_{n-1}\right) d x_{i} d x_{i+1} \ldots d x_{n-1} \\
& \quad=\int_{X_{i}(f)}^{1} \int_{0}^{1} \ldots \int_{0}^{1} V_{f}\left(X_{1}(f), \ldots, X_{i-1}(f), x_{i}, \ldots, x_{n-1}\right) d x_{i} d x_{i+1} \ldots d x_{n-1} .
\end{aligned}
$$

Пусть $x_{i}^{0}=X_{i}(f)$. Из условия (ii) следует, что определение отображения $X_{i}(f)$ корректно и множество $Y_{\varepsilon, L}\left(f, x_{1}^{0}, \ldots, x_{n-1}^{0}\right)$ непусто. Для $\left(x_{1}, \ldots, x_{n-1}\right) \in D$ и $0 \leqslant j \leqslant m$ определим отображение $Z_{j}: C[0,1] \times D \rightarrow \mathbb{R}$ по формуле

$$
Z_{j}\left(f, x_{1}, \ldots, x_{n-1}\right)=\int_{Y_{\varepsilon, L}\left(f, x_{1}, \ldots, x_{n-1}\right)} \ldots \int_{\mu\left(Y_{\varepsilon, L}\left(f, x_{1}, \ldots, x_{n-1}\right)\right)} .
$$

Пусть набор $\left(y_{0}^{0}, y_{1}^{0}, \ldots, y_{m}^{0}\right)$ является центром масс множества $Y_{\varepsilon, L}\left(f, x_{1}^{0}, \ldots\right.$, $\left.x_{n-1}^{0}\right), y_{j}^{0}=Z_{j}\left(f, x_{1}^{0}, \ldots, x_{n-1}^{0}\right)$. Тогда отображение $G: C[0,1] \rightarrow L, G(f)=$ $F\left(x_{1}^{0}, \ldots, x_{n-1}^{0}, y_{0}^{0}, \ldots, y_{m}^{0}\right)$, является непрерывной $\varepsilon$-выборкой на множество $L$. Действительно, тот факт, что отображение $G$ есть $\varepsilon$-выборка, вытекает из определения отображения $G$ и условия (i). Доказательство непрерывности $G$ может быть проведено с использованием условия (iii).

Через $S_{n}^{d}$ обозначим множество всех непрерывных кусочно-полиномиальных функций $l \in C[0,1]$, т. е. функций, для которых найдется такое разбиение $0=$ $x_{0}<x_{1}<\cdots<x_{n-1}<x_{n}=1$, что $\left.l\right|_{\left[x_{i-1}, x_{i}\right]}$ является полиномом степени $\leqslant d$ для всех $i, 1 \leqslant i \leqslant n$. Отметим, что $S_{n}^{d}$ является множеством существования, но не является множеством единственности при $n \geqslant 2$. Из теоремы 1 можно вывести такие следствия:

СледствиЕ 1. Для любых $\varepsilon>0 u n>1$ существует непрерывная $\varepsilon$-выборка на множество $S_{n}^{1}$. 
Для доказательства следствия 1 достаточно рассмотреть множество $D=\left\{\left(x_{1}\right.\right.$, $\left.\left.\ldots, x_{n-1}\right): 0<x_{1}<\cdots<x_{n-1}<1\right\}$ и отображение $F$, сопоставляющее точке $\left(x_{1}, \ldots, x_{n-1}\right) \in D$ и точке $\left(y_{0}, \ldots, y_{n}\right) \in \mathbb{R}^{n+1}$ ломаную с вершинами в $\left(0, y_{0}\right)$, $\left(x_{1}, y_{1}\right), \ldots,\left(x_{n-1}, y_{n-1}\right),\left(1, y_{n}\right)$, и проверить, что $S_{n}^{1}=L=\operatorname{Im} F \in K$.

Из теоремы 1 также можно вывести следующий результат Конягина [2].

СлЕДСтвиЕ 2. Для любого $\varepsilon>0$ и произвольных целых $m \geqslant 0 u n \geqslant 0$ существует непрерывная в-выборка на множество $R_{m, n}$.

ТЕОРема 2. Ни при каких $n, d>1 u \varepsilon \geqslant 0$ не существует непрерьвной

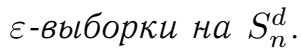

Укажем основные идеи доказательства теоремы 2 для $n=2, d \geqslant 2$. Обозначим через $\|\cdot\|_{\Delta}$ равномерную норму на отрезке $\Delta$. Пусть $\varepsilon=(100 D)^{-2}$, где $D=$ $\sup \left\{\|P\|_{[0,2]}: P=a_{d} x^{d}+\cdots+a_{0},(-1)^{i} P(i /(2 d+1))<1, i \in\{0, \ldots, 2 d+1\}\right\}$. Рассмотрим $h_{1}, h_{2} \in C[0,1]$, такие, что $\left.h_{1}\right|_{[0,2 / 3]}=0,\left.h_{1}\right|_{[2 / 3,1]}=\left(x-\frac{1}{3}\right)\left(x-\frac{2}{3}\right)$ и $\left.h_{2}\right|_{[0,1 / 3]}=0,\left.h_{2}\right|_{[1 / 3,1]}=\left(x-\frac{1}{3}\right)\left(x-\frac{2}{3}\right)$. Положим для $g_{1}, g_{2} \in C[0,1]$

$$
\begin{gathered}
M\left(g_{1}, g_{2}\right)=\left\{f \in C[0,1]:\|f\|_{[1 / 3,2 / 3]} \leqslant 5,\left\|f-g_{1}\right\|_{[0,1 / 3]}=\left\|f-g_{2}\right\|_{[2 / 3,1]}=7\right. \text { и } \\
(-1)^{i-1}\left(f\left(\frac{i}{3(2 d+1)}\right)-g_{1}\left(\frac{i}{3(2 d+1)}\right)\right)=(-1)^{i-1}\left(f\left(\frac{2}{3}+\frac{i}{3(2 d+1)}\right)\right. \\
\left.\left.-g_{2}\left(\frac{2}{3}+\frac{i}{3(2 d+1)}\right)\right)=7 \text { при } i \in\{0, \ldots 2 d+1\}\right\} .
\end{gathered}
$$

Предположим, что $G-$ непрерывная $\varepsilon$-выборка. Рассмотрим $f_{0} \in M\left(h_{1}, h_{2}\right)$; тогда или $\left\|G\left(f_{0}\right)-h_{1}\right\|_{[2 / 5,3 / 5]}<\varepsilon D$, или $\left\|G\left(f_{0}\right)-h_{2}\right\|_{[2 / 5,3 / 5]}<\varepsilon D$. В первом случае (второй случай разбирается аналогично) рассмотрим отображение $A \in$ $C([0,1], C[0,1])$, такое, что $A(0)=f_{0}$ и $A(t) \in M\left(\varepsilon^{1 / 2} t\left(x-\frac{1}{3}\right) x, h_{2}\right)$. Заметим, что $\rho\left(A(1), S_{n}^{d}\right)=7$ и или $\left\|G(A(1))-h_{2}\right\|_{[2 / 5,3 / 5]}<\varepsilon D$, или $\| G(A(1))-\varepsilon^{1 / 2} x(x-$ $\left.\frac{1}{3}\right) \|_{[2 / 5,3 / 5]}<\varepsilon D$. Последнее невозможно, так как в этом случае $G(A(1))\left(\frac{2}{3}\right)-$ $A(1)\left(\frac{2}{3}\right) \geqslant 7+\frac{2}{9} \varepsilon^{1 / 2}-\varepsilon D>\rho\left(A(1), S_{n}^{d}\right)+\varepsilon$. Отсюда вытекает, что множество $\left\{G(A(t))\left(\frac{1}{2}\right): t \in[0,1]\right\}$ несвязно в $\mathbb{R}$. Следовательно, $G(A(t))$ имеет разрыв на $[0,1]$, что противоречит непрерывности $\varepsilon$-выборки $G$. Доказательство теоремы для произвольного $\varepsilon$ дает соответствующая гомотетия.

Похожая конструкция может дать доказательство прямой импликации в следующей теореме.

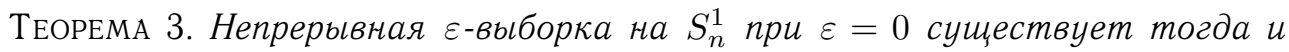
только тогда, когда $n=1$ или $n=2$.

Автор выражает благодарность И. Г. Царькову, М. И. Карлову и А. Р. Алимову за помощь, оказанную при написании этой статьи.

\section{ЛИТЕРАТУРА}

1. Чебышёв П. Л. В кн.: Сочинения, т. II, 1859, с. 151-235. 2. Конягин С. В. Матем. заметки, 44, № 3, 404 (1988). 3. Maehly H., Witzgall Ch. Num. Math., 2, No. 5, 293-307 (1960). 